\title{
MODULI OF CONTINUITY OF HARMONIC QUASIREGULAR MAPPINGS ON BOUNDED DOMAINS
}

\author{
Ali Abaob, Miloš Arsenović, Miodrag Mateljević and Abejela Shkheam \\ University of Belgrade, Faculty of Mathematics \\ Studentski Trg 16, 11000 Belgrade, Serbia; elhrarya@yahoo.com \\ University of Belgrade, Faculty of Mathematics \\ Studentski Trg 16, 11000 Belgrade, Serbia; arsenovic@matf.bg.ac.rs \\ University of Belgrade, Faculty of Mathematics \\ Studentski Trg 16, 11000 Belgrade, Serbia; miodrag@matf.bg.ac.rs \\ University of Belgrade, Faculty of Mathematics \\ Studentski Trg 16, 11000 Belgrade, Serbia; abejelashkhea@yahoo.com
}

\begin{abstract}
We prove that $\omega_{u}(\delta) \leq C \omega_{f}(\delta)$, where $u: \bar{\Omega} \rightarrow \mathbf{R}^{n}$ is the harmonic extension of a continuous map $f: \partial \Omega \rightarrow \mathbf{R}^{n}$, if $u$ is a $K$-quasiregular map and $\Omega$ is bounded in $\mathbf{R}^{n}$ with $C^{2}$ boundary. Here $C$ is a constant depending only on $n, \omega_{f}$ and $K$ and $\omega_{h}$ denotes the modulus of continuity of $h$. We also prove a version of this result for $\Lambda_{\omega}$-extension domains with $c$-uniformly perfect boundary and quasiconformal mappings.
\end{abstract}

\section{Introduction}

Let $\Omega \subset \mathbf{R}^{n}$ be a domain (connected, non-empty, open set). Harmonic quasiregular (briefly, hqr) mappings in the plane were studied first by Martio in [20], for a review of this subject and further results see [22] and references cited there. Moduli of continuity of harmonic quasiregular mappings in $\mathbf{B}^{n}$ were studied by several authors, see $[15,11,2]$. In this paper, our main goal is to extended one of the main results from [1] to more general domains in $\mathbf{R}^{n}$. In fact, the following theorem was proved in [1].

Theorem 1. If $u: \overline{\mathbf{B}}^{n} \rightarrow \mathbf{R}^{n}$ is a continuous map which is $K$-quasiregular and harmonic in $\mathbf{B}^{n}$, then $\omega_{u}(\delta) \leq C \omega_{f}(\delta)$ for $\delta>0$, where $f=\left.u\right|_{S^{n-1}}$ and $C$ is a constant depending only on $K, \omega_{f}$ and $n$.

We use two methods to extend this result. The first method is to use the following theorem from [1].

Theorem 2. There is a constant $q=q(K, n) \in(0,1)$ such that $|u|^{q}$ is subharmonic in $\Omega \subset \mathbf{R}^{n}$ whenever $u: \Omega \rightarrow \mathbf{R}^{n}$ is a $K$-quasiregular harmonic map.

The above theorem combined with Poisson integral representation gives Theorem 1. The main point is that a similar argument can be carried out without using explicit formula for the Poisson kernel. In fact suitable estimates are sufficient, and these rely on pointwise estimates for the Poisson kernel which are available in the

doi:10.5186/aasfm.2013.3848

2010 Mathematics Subject Classification: Primary 30C80, 30C62; Secondary 30C55, 30H05.

Key words: Lipschitz-type spaces, harmonic mappings, quasiregular mappings, uniform domains. 
case of bounded domain $\Omega \subset \mathbf{R}^{n}$ with $C^{2}$ boundary, see [17, 18]. We prove a version of Theorem 1 for domains $\Omega$ with $C^{2}$ boundary, see Theorem 4 below.

The second method is essentially based on a capacity estimate of Martio and Näkki [21]. Let us introduce needed terminology and notation.

Throughout this paper $\Omega \subset \mathbf{R}^{n}$ is bounded domain, $\delta(x)=\operatorname{dist}\left(x, \Omega^{c}\right)$ and $B_{x}=B(x, \delta(x) / 2)$ for $x \in \Omega$. If $\Omega$ has $C^{2}$ boundary, then $P_{\Omega}$ denotes the Poisson kernel for $\Omega$.

Given a subset $E$ of $\mathbf{C}^{n}$ or $\mathbf{R}^{n}$; a function $f: E \rightarrow \mathbf{C}$ (or, more generally, a mapping $f$ from $E$ into $\mathbf{C}^{m}$ or $\mathbf{R}^{m}$ ) is said to belong to the Lipschitz space $\Lambda_{\omega}(E)$ if there is a constant $L=L(f)=L(f ; E)$ such that

$$
|f(x)-f(y)| \leq L \omega(|x-y|)
$$

for all $x, y \in E$, or equivalently, $\omega_{f}(|x-y|) \leq L \omega(|x-y|)$ for $x, y \in E$. Here $\omega:[0,+\infty) \rightarrow[0,+\infty)$ is a majorant in the sense of Hinkkanen, see [10], which means $\omega$ is non-decreasing and $\omega(2 t) \leq 2 \omega(t)$. In that case we also say that $f$ is $\omega$-Lipschitz function. We remark that $\omega$ need not be continuous, that we may have $\omega(0)>0$ and that $\omega(A t) \leq A \omega(t)$ for all $t \geq 0$ and $A \geq 1$. The most important special case is $\omega_{\alpha}(t)=t^{\alpha}, 0<\alpha \leq 1$, when we get classical concept of Lipschitz or Hölder continuity.

There has been much work on Lipschitz-type properties of quasiconformal mappings. This topic was treated, among many other papers, in [6].

Following [8] and [19], we say that a function $f$ belongs to the local Lipschitz space loc $\Lambda_{\omega}(\Omega, L)$ if (1) holds, with a fixed $L \geq 0$, whenever $x \in \Omega$ and $y \in B_{x}$. We set $\operatorname{loc} \Lambda_{\omega}(\Omega)=\cup_{L \geq 0} \operatorname{loc} \Lambda_{\omega}(\Omega, L)$. If $\omega(t)=t^{\alpha}, 0<\alpha \leq 1$, we use notation $\Lambda_{\alpha}(\Omega)$, $\operatorname{loc} \Lambda_{\omega}(\Omega)$ and $\operatorname{loc} \Lambda_{\alpha}(\Omega, L)$.

A domain $\Omega$ is a $\Lambda_{\omega}$-extension domain if $\Lambda_{\omega}(\Omega)=\operatorname{loc} \Lambda_{\omega}(\Omega)$.

A compact set $E$ in $\mathbf{R}^{n}$ is called $c$-uniformly perfect, $0<c<1$, if $E$ contains at least two points and if for each $x \in E$ and $0<r<\operatorname{diam}(E)$, the spherical ring $B(x, r) \backslash \bar{B}(x, c r)$ meets $E$.

If $V$ is a subset of $\mathbf{R}^{n}$ and $u: V \rightarrow \mathbf{R}^{m}$, we define

$$
\operatorname{osc}_{V} u=\sup \{|u(x)-u(y)|: x, y \in V\} .
$$

For $\Omega \subset \mathbf{R}^{n}$ let $O C^{1}(\Omega)$ denote the class of all $f \in C^{1}\left(\Omega, \mathbf{R}^{n}\right)$ such that

$$
\delta(x)\left|f^{\prime}(x)\right| \leq C \operatorname{osc}_{B_{x}} f, \quad x \in \Omega .
$$

We denote by $O C^{2}(\Omega)$ the class of all $f \in C^{2}\left(\Omega, \mathbf{R}^{n}\right)$ such that for some constant $C$ we have

$$
\sup _{B_{x}} \delta^{2}(x)|\Delta f(x)| \leq C \operatorname{Osc}_{B_{x}} f, \quad x \in \Omega .
$$

We remind the reader that $B_{x}$ denotes $B(x, \delta(x) / 2)$. It was observed in [23] that $O C^{2}(\Omega) \subset O C^{1}(\Omega)$. Note that every harmonic mapping $f: \Omega \rightarrow \mathbf{R}^{n}$ is in $O C^{2}(\Omega)$.

We also show that under some conditions a function $f \in O C^{2}(\Omega)$ is $\omega$-Lipschitz function on $\Omega$ if and only if it satisfies Hardy-Littlewood $(C, \omega)$-property:

$$
\delta(x)\left|f^{\prime}(x)\right| \leq C \omega(\delta(x)), \quad x \in \Omega .
$$

Relying on this characterization and a result from [3] (Lemma 2 below) we also prove a version of Theorem 1 for $\Lambda_{\omega}$-extension domain with $c$-uniformly perfect boundary and quasiconformal mappings, where $\omega$ is a majorant. 
Finally, we give a simple proof of Dyakonov's result on relation between moduli of continuity of $|f|$ and $f$, see $[6]$.

We follow the usual convention, letter $C$ denotes a constant that can change its value from one occurrence to the next.

\section{Auxiliary results}

Let $\Omega \subset \mathbf{R}^{n}$ be a bounded domain with $C^{2}$ boundary. Clearly, an explicit formula for the Poisson kernel is available only in special cases, like the ball. However, it is possible to obtain an asymptotic formula for the Poisson kernel on smoothly bounded domains. The standard asymptotic formula, see [16] or [25], is

$$
P_{\Omega}(x, y) \asymp \frac{\delta(x)}{|x-y|^{n}}
$$

for $x \in \Omega$ and $y \in \partial \Omega$. The following theorem was proved in [17].

Theorem 3. Let $\Omega \subset \mathbf{R}^{n}$ be a bounded domain with $C^{2}$ boundary. Let $P_{\Omega}: \Omega \times$ $\partial \Omega \rightarrow \mathbf{R}^{+}$be the Poisson kernel for $\Omega$. Then there are constants $C_{1}, C_{2}>0$ such that

$$
C_{1} \frac{\delta(x)}{|x-y|^{n}} \leq P_{\Omega}(x, y) \leq C_{2} \frac{\delta(x)}{|x-y|^{n}}
$$

The following technical lemma is used in the next section when we consider smoothly bounded domains.

Lemma 1. Assume $\Omega$ has $C^{1}$ boundary. Then there is a constant $C$ depending only on $\Omega$, such that

$$
\operatorname{area}\left(\partial \Omega \cap B\left(z_{0}, r\right)\right) \leq C r^{n-1}
$$

for all $r>0$ and all $z_{0} \in \partial \Omega$.

Proof. We have a local parametrization of $\partial \Omega$ :

$$
x_{1}=x_{1}\left(u_{1}, u_{2}, \ldots, u_{n-1}\right), x_{2}=x_{2}\left(u_{1}, u_{2}, \ldots, u_{n-1}\right), \ldots, x_{n}=x_{n}\left(u_{1}, u_{2}, \ldots, u_{n-1}\right) \text {, }
$$

i.e., $x=x(u), x=\left(x_{1}, x_{2}, \ldots, x_{n}\right)$, where $x_{j} \in C^{1}(U), U \subset \mathbf{R}^{n-1}$.

Since $\partial \Omega$ is compact, it suffices to prove the estimate (5) for $z_{0} \in x(K)$, where $K \subset U$ is compact. Now fix a compact $K \subset U$. We have, for any measurable $S \subset K$ :

$$
\operatorname{area}(x(S))=\int_{S} \sqrt{g} d u
$$

where $g=\operatorname{det}\left(g_{i j}\right)_{i, j=1}^{n-1}, \quad g_{i j}=\sum_{m=1}^{n} \frac{\partial x_{m}}{\partial u_{i}} \frac{\partial x_{m}}{\partial u_{j}}$.

Note that $g_{i j} \in C(U)$, so $\sqrt{g}$ is a strictly positive continuous function on $U$. Therefore $0<c \leq \sqrt{g} \leq C<+\infty$ on $K$.

Let $u_{1}, u_{2} \in K$ and $z_{1}=x\left(u_{1}\right), z_{2}=x\left(u_{2}\right)$. Since $x=x(u)$ is a parametrization, we have

$$
\left|z_{1}-z_{2}\right| \asymp\left|u_{1}-u_{2}\right| .
$$


Setting $z_{0}=x\left(u_{0}\right)$ and using (6), we see that there is a constant $M$ such that

$$
\begin{aligned}
& \operatorname{area}\left(\partial \Omega \cap B\left(z_{0}, r\right)\right) \leq \operatorname{area}\left(x\left(B\left(u_{0}, M r\right)\right)\right)=\int_{B\left(u_{0}, M r\right)} \sqrt{g} d u \\
& \leq C \int_{B\left(u_{0}, M r\right)} d u \leq C \operatorname{Vol}_{n-1}\left(B\left(u_{0}, M r\right)\right)=C(M r)^{n-1}=C r^{n-1} .
\end{aligned}
$$

\section{The case of $C^{2}$ boundary}

In this section we follow the first method described in the introduction and obtain the following generalization of Theorem 1.

Theorem 4. Let $\Omega \subset \mathbf{R}^{n}$ be a bounded domain with $C^{2}$ boundary, and assume $u: \bar{\Omega} \rightarrow \mathbf{R}^{n}$ is a continuous map which is $K$-quasiregular and harmonic in $\Omega$, then $\omega_{u}(\delta) \leq C \omega_{f}(\delta)$ for $\delta>0$, where $f=\left.u\right|_{\partial \Omega}$ and $C$ is a constant depending only on $K, \omega_{f}$ and $n$.

Proof. The proof is similar to the proof in [1], but with additional technical difficulties due to the lack of an explicit formula for $P_{\Omega}$. Instead we rely on Lemma 1 and crucial estimates (4), using a dyadic decomposition of $\partial \Omega$.

Let us recall some properties of $\omega_{f}$ :

$$
\omega_{f}\left(\delta_{1}+\delta_{2}\right) \leq C\left(\omega_{f}\left(\delta_{1}\right)+\omega_{f}\left(\delta_{2}\right)\right), \quad \omega_{f}(\lambda \delta) \leq C \lambda \omega_{f}(\delta)
$$

are valid for $\delta, \delta_{1}, \delta_{2}>0$ and $\lambda \geq 1$. First, fix an exponent $q=q(K, n)<1$ from Theorem 2. Fix $w \in \partial \Omega$ and $z \in \Omega$. Then $\varphi(\xi)=|u(w)-u(\xi)|^{q}$ is subharmonic in $\Omega$ and therefore we have

$$
\varphi(z) \leq \int_{\partial \Omega} P_{\Omega}(z, \xi) \varphi(\xi) d \sigma(\xi)
$$

But, for $\xi \in \partial \Omega$ we have

$$
\begin{aligned}
\varphi(\xi) & =|u(w)-u(\xi)|^{q} \leq \omega_{f}(|w-\xi|)^{q} \leq \omega_{f}(|w-z|+|z-\xi|)^{q} \\
& \leq C\left[\omega_{f}(|w-z|)^{q}+\omega_{f}(|z-\xi|)^{q}\right],
\end{aligned}
$$

and integration against Poisson kernel gives

$$
\varphi(z) \leq C\left[\omega_{f}(|w-z|)^{q}+\int_{\partial \Omega} P_{\Omega}(z, \xi) \omega_{f}(|z-\xi|)^{q} d \sigma(\xi)\right] .
$$

Let $z_{0} \in \partial \Omega$ be the closest point on the boundary to $z \in \Omega$. Then

$$
|z-\xi| \asymp \delta(z)+\left|z_{0}-\xi\right|
$$

for $\xi \in \partial \Omega$. Therefore

$$
\omega_{f}(|z-\xi|) \leq C \omega_{f}\left(\delta(z)+\left|z_{0}-\xi\right|\right) \leq C \frac{\delta(z)+\left|z_{0}-\xi\right|}{\delta(z)} \omega_{f}(\delta(z)) .
$$

By Theorem 3 we get

$$
\varphi(z) \leq C \omega_{f}(|w-z|)^{q}+C \int_{\partial \Omega}(\delta(z))^{1-q} \frac{\left(\delta(z)+\left|z_{0}-\xi\right|\right)^{q}}{|z-\xi|^{n}} d \sigma(\xi) \omega_{f}(\delta(z))^{q} .
$$


Next we prove that the integral appearing above is bounded as a function of $z \in \Omega$. Set $\delta(z)=\delta$. Since $|z-\xi| \geq C\left(\delta(z)+\left|z_{0}-\xi\right|\right)$, we get

$$
\int_{\partial \Omega}(\delta(z))^{1-q} \frac{\left(\delta(z)+\left|z_{0}-\xi\right|\right)^{q}}{|z-\xi|^{n}} d \sigma(\xi) \leq C \delta^{1-q} \int_{\partial \Omega}\left(\delta+\left|z_{0}-\xi\right|\right)^{q-n} d \sigma(\xi) .
$$

Now, we use the following decomposition of $\partial \Omega$ : $\partial \Omega=\bigcup_{k=0}^{\infty} M_{k}$ where

$$
M_{k}=\left\{\xi \in \partial \Omega: 2^{k-1} \delta \leq d\left(\xi, z_{0}\right)<2^{k} \delta\right\}, \quad k \geq 1,
$$

and

$$
M_{0}=\left\{\xi \in \partial \Omega: d\left(\xi, z_{0}\right)<\delta\right\} .
$$

Using Lemma 1 we obtain:

$$
\begin{aligned}
& \int_{\partial \Omega}(\delta(z))^{1-q} \frac{\left(\delta(z)+\left|z_{0}-\xi\right|\right)^{q}}{|z-\xi|^{n}} d \sigma(\xi) \leq C \delta^{1-q} \sum_{k=0}^{\infty} \int_{M_{k}}\left(\delta+\left|z_{0}-\xi\right|\right)^{q-n} d \sigma(\xi) \\
& \leq C \delta^{1-q} \sum_{k=0}^{\infty} \int_{M_{k}}\left(2^{k} \delta\right)^{q-n} d \sigma(\xi) \leq C \sum_{k=0}^{\infty} 2^{k(q-n)} \delta^{1-n} \text { area }\left(M_{k}\right) \\
& \leq C \sum_{k=0}^{\infty} 2^{k(q-n)} \delta^{1-n}\left(2^{k} \delta\right)^{n-1} \leq C \sum_{k=0}^{\infty} 2^{k(q-1)}<+\infty .
\end{aligned}
$$

Note that here we used $q \in(0,1)$. Hence we get

$$
\varphi(z) \leq C\left[\omega_{f}(|w-z|)^{q}+\omega_{f}(\delta(z))^{q}\right] \leq C \omega_{f}(|w-z|)^{q},
$$

and therefore we proved

$$
|u(w)-u(z)| \leq C \omega_{f}(|w-z|) \quad \text { for } w \in \partial \Omega, z \in \Omega .
$$

In view of Lemma A.1. from [5] this estimate suffices to complete the proof.

If we assume that $f$ is quasiconformal, then we can significantly relax the $\mathrm{C}^{2}$ assumption on the boundary, see Theorem 5 below.

\section{The case of uniformly perfect boundary}

In this section we work with much more general domains, but here we consider only quasiconformal harmonic (or more general $O C^{2}$ ) mappings.

Proposition 1. Let $f \in C^{1}\left(\Omega, \mathbf{R}^{n}\right)$ and let $\omega$ be a continuous majorant such that $\omega_{*}(t)=\omega(t) / t$ is non-increasing for $t>0$. Assume $f$ satisfies the following property:

$$
\delta(x)\left|f^{\prime}(x)\right| \leq C \omega(\delta(x)), \quad x \in \Omega, \quad(H L(\omega, C)),
$$

which we call Hardy-Littlewood $(C, \omega)$-property. Then

$$
f \in \operatorname{loc} \Lambda_{\omega}(L ; \Omega), \quad\left(\operatorname{loc} \Lambda_{\omega}\right) .
$$

If, in addition, $f$ is harmonic in $\Omega$ or, more generally, $f \in O C^{2}(\Omega)$, then $(H L(\omega, C))$ is equivalent with $\left(\operatorname{loc} \Lambda_{\omega}\right)$. 
Proof. Let us prove that $(H L(\omega, C))$ implies $\left(\operatorname{loc} \Lambda_{\omega}\right)$. If $y \in B_{x}$, then

$$
|f(y)-f(x)| \leq \int_{[x, y]}\left|f^{\prime}(z)\right| d s(z) \leq|y-x| \max _{z \in[x, y]}\left|f^{\prime}(z)\right| \leq C|y-x| \max _{z \in[x, y]} \frac{\omega(\delta(z))}{\delta(z)} .
$$

Now, for every $z \in[x, y] \subset B_{x}$ we have $|x-y| \leq \delta(x) / 2 \leq \delta(z)$, and since $\omega_{\star}$ is non-increasing we get $\omega(\delta(z)) / \delta(z) \leq \omega(|x-y|) /|x-y|$. This, combined with the above estimate, gives $|f(y)-f(x)| \leq C \omega(|y-x|)$.

Next we assume $f: \Omega \rightarrow \mathbf{R}^{n}$ is a harmonic mapping in $\operatorname{loc} \Lambda_{\omega}(\Omega, L)$. We set $M_{x}(r)=\max \{|f(y)|:|y-x|=r\}$ for $x \in \Omega, 0 \leq r<\delta(x)$. Since $f$ is harmonic we have $r\left|f^{\prime}(x)\right| \leq C_{n} M_{x}(r)$ and $f \in \operatorname{loc} \Lambda_{\omega}(\Omega, L)$ implies $M_{x}(r) \leq L \omega(r)$. Therefore $r\left|f^{\prime}(x)\right| \leq C_{n} L \omega(r)$ for $0<r<\delta(x)$. Letting $r \rightarrow \delta(x)$ we deduce $(H L(\omega, C))$ with $C=C_{n} L$.

Now we give a proof for the more general case of $f \in O C^{2}(\Omega)$. We assume $f \in \operatorname{loc} \Lambda_{\omega}(L, \Omega)$. Let us choose $x \in \Omega$. Since $\operatorname{diam} B_{x}=\delta(x)$, we have

$$
\sup _{B_{x}} \delta^{2}(y)|\Delta f(y)| \leq \operatorname{osc}_{B_{x}} f \leq L \omega(\delta(x)) .
$$

Since $\delta(y) \asymp \delta(x)$ for $y \in B_{x}$ the above estimate gives

$$
|\Delta f(y)| \leq C L \frac{\omega(\delta(x))}{\delta^{2}(x)}, \quad y \in B_{x} .
$$

Next we use gradient estimates for Poisson equation in the ball $B_{x}$, see Theorem 3.9 from [9], and obtain

$$
\left|f^{\prime}(x)\right| \leq C\left[\frac{1}{\delta(x)} \sup _{\partial B_{x}}|f|+\delta(x) \sup _{B_{x}}|\Delta f|\right] .
$$

Since both $f^{\prime}$ and $\Delta f$ do not change if we replace $f$ with $f-f(x)$ we see that

$$
\left|f^{\prime}(x)\right| \leq C\left[\frac{1}{\delta(x)} \operatorname{osc}_{\partial B_{x}}|f|+\delta(x) \sup _{B_{x}}|\Delta f|\right] \leq C L \frac{\omega(\delta(x))}{\delta(x)} .
$$

Note that a similar argument appeared in [23], it was used to prove inclusion $O C^{2}(\Omega) \subset$ $O C^{1}(\Omega)$.

An immediate consequence of the above proposition is the following corollary.

Corollary 1. Let $\omega$ be a continuous majorant such that $\omega_{\star}(t)=\omega(t) / t$ is nonincreasing for $t>0$ and let $\Omega \subset \mathbf{R}^{n}$ be a domain which has $\Lambda_{\omega}$-extension property. Then an $O C^{2}$ mapping (in particular, a harmonic mapping) $f: \Omega \rightarrow \mathbf{R}^{n}$ belongs to $\Lambda_{\omega}(\Omega)$ if and only if it has Hardy-Littlewood $(C, \omega)$-property.

Remark 1. If the mapping $f$ in the Proposition 1 and Corollary 1 extends continuously to $\bar{\Omega}$, then the assumption of continuity of $\omega$ can be omitted.

Theorem 4 can be restated: Let $\Omega \subset \mathbf{R}^{n}$ be a bounded domain with $C^{2}$ boundary. Assume $u: \bar{\Omega} \rightarrow \mathbf{R}^{n}$ is a continuous map which is $K$-quasiregular map and harmonic in $\Omega$, and $f \in \Lambda_{\omega}(\partial \Omega ; L)$ where $f=\left.u\right|_{\partial \Omega}$. Then

$$
u \in \Lambda_{\omega}(\Omega ; C L), \quad C=C\left(K, \omega_{f}, n, \Omega\right) .
$$

If $\Omega$ is a $\Lambda_{\omega}$-extension domain, then (7) is equivalent to Hardy-Littlewood ( $\left.\omega, C_{1}\right)$ property: $\delta(x)\left|f^{\prime}(x)\right| \leq C_{1} \omega(\delta(x))$ for all $x \in \Omega$. 
Special cases of this result, for the disk and unit ball and holomorphic functions are well know as Privalov theorem. Hardy-Littlewood theorem is concerned by characterization of Lipschitz spaces in terms of growth of derivative.

The following result is contained in Theorem 3.2 from [3].

Lemma 2. Let the boundary of a bounded domain $\Omega$ in $\mathbf{R}^{n}$ be c-uniformly perfect. If $f$ is a continuous mapping of $\bar{\Omega}$ into $\mathbf{R}^{n}$ which is quasiconformal in $\Omega$ and if

$$
|f(x)-f(y)| \leq \omega(|x-y|)
$$

for all $x, y \in \partial \Omega$ and for some majorant $\omega$, then

$$
|f(x)-f(y)| \leq C \omega(|x-y|)
$$

for all $y \in \partial \Omega$ and $x \in \Omega$, where $C$ depends only on $c, n, K(f)$ and $\operatorname{diam}(\Omega)$.

Using Lemma 2 we prove the following generalization of Theorem 1.

Theorem 5. Let the boundary of a bounded domain $\Omega$ in $\mathbf{R}^{n}$ be c-uniformly perfect. Assume $f$ is a continuous mapping of $\bar{\Omega}$ into $\mathbf{R}^{n}$ which is quasiconformal in $\Omega$ and

$$
|f(x)-f(y)| \leq \omega(|x-y|), \quad x, y \in \partial \Omega,
$$

for some majorant $\omega$. Assume one of the following two conditions is satisfied:

a) $f$ is harmonic in $\Omega$.

b) $f \in O C^{2}(\Omega), \omega(t) / t$ is non increasing for $t>0$ and $\Omega$ is an $\Lambda_{\omega}$-extension domain.

Then the following estimate holds:

$$
|f(x)-f(y)| \leq C \omega(|x-y|), \quad x, y \in \Omega .
$$

Proof. Let us assume $f$ is harmonic. By Lemma 2, estimate (11) holds for all $x \in \partial \Omega$ and all $y \in \Omega$. Using Lemma A.1. from [5] we deduce that the same estimate is valid for all $x, y \in \Omega$.

Now we consider condition b). Fix a point $x \in \Omega$. Choose a point $\xi \in \partial \Omega$ such that $|x-\xi|=\delta(x)$ and set $f_{0}(z)=f(z)-f(\xi), z \in \bar{\Omega}$. We employ again gradient estimates for the Poisson equation, as in the proof of Proposition 1. Since $f^{\prime}=f_{0}^{\prime}$ and $\Delta f=\Delta f_{0}$ we obtain

$$
\left|f^{\prime}(x)\right| \leq C_{n}\left[\frac{1}{\delta(x)} \sup _{\partial B_{x}}\left|f_{0}\right|+\delta(x) \sup _{B_{x}}|\Delta f|\right] .
$$

However, since $B_{x} \subset B(\xi, 3 \delta(x) / 2)$, Lemma 2 gives

$$
\sup _{\partial B_{x}}\left|f_{0}(z)\right| \leq \sup _{\partial B_{x}}|f(z)-f(\xi)| \leq C \omega(3 \delta(x) / 2) \leq C \omega(\delta(x)) .
$$

Also, $O C^{2}$ condition gives $\sup _{B_{x}}|\Delta f| \leq C \delta^{-2}(x)$. These estimates, combined with (12) give $\left|f^{\prime}(x)\right| \leq C \omega(\delta(x)) / \delta(x)$. Hence we proved that $f$ has Hardy-Littlewood $(C, \omega)$ property. Now the result follows from Corollary 1.

4.1. Dyakonov's result. Now we give a simple proof of a Dyakonov's result from [6] which relates moduli of continuity of $f$ and $|f|$ in the special case of quasiconformal $f$. 
Our proof is based on distortion property of quasiconformal mappings (see [7, p. 383], [26, p. 63]):

$$
\bar{B}\left(f(x), c_{*} \delta_{*}(x)\right) \subset f\left(B_{x}\right) \subset B\left(f(x), C_{*} \delta_{*}(x)\right), \quad x \in \Omega,
$$

for a $K$-quasiconformal mapping $f: \Omega \rightarrow f(\Omega)=\Omega^{\prime}$, where $\delta_{*}(x)=\operatorname{dist}\left(f(x), \partial \Omega^{\prime}\right)$. The constants $C_{*}$ and $c_{*}$ depend on $n$ and $K$ only.

Theorem 6. Suppose $f: \Omega \rightarrow f(\Omega)=\Omega^{\prime}$ is quasiconformal in domain $\Omega \subset \mathbf{R}^{n}$. Let $0<\alpha \leq 1$. If $|f| \in \operatorname{loc} \Lambda_{\alpha}(\Omega, L)$, then $f \in \operatorname{loc} \Lambda_{\alpha}(\Omega, C L)$.

If, in addition, $\Omega$ is a $\Lambda_{\alpha}$-extension domain, then $f \in \Lambda_{\alpha}(\Omega)$.

Proof. Let us choose $x \in \Omega$ and set $R(x)=c_{*} \delta_{*}(x)$. We first prove the following:

$$
\exists x_{1}, x_{2} \in B_{x}:\left|f\left(x_{1}\right)\right|-\left|f\left(x_{2}\right)\right| \geq R(x) .
$$

Let $l$ be the line passing through 0 and $f(x)$, it intersects the sphere $\partial B(f(x), R(x))$ at points $y_{1}$ and $y_{2}$. By the first inclusion in (13) these two points lie in $f\left(B_{x}\right)$, hence $x_{k}=f^{-1}\left(y_{k}\right) \in B_{x}, k=1,2$. We consider two cases:

a) If $0 \notin B(f(x), R(x))$ and $\left|y_{2}\right| \geq\left|y_{1}\right|$, then $\left|y_{2}\right|-\left|y_{1}\right|=2 R(x)$.

b) If $0 \in B(f(x), R(x))$, then for example $0 \in\left[y_{1}, f(x)\right]$ and if we choose $x_{1}=x$, we find $\left|y_{2}\right|-|f(x)|=R(x)$ and this yields (14).

Now we obtain, using (14), that

$$
c_{*} \delta_{*}(x)=R(x) \leq\left|f\left(x_{1}\right)\right|-\left|f\left(x_{2}\right)\right| \leq L\left|x_{1}-x_{2}\right|^{\alpha} \leq L \delta(x)^{\alpha} .
$$

Using the second inclusion in (13) we obtain

$$
\left|f\left(z_{1}\right)-f\left(z_{2}\right)\right| \leq 2 C_{*} \delta_{*}(x) \leq 2 \frac{C_{*}}{c_{*}} L \delta(x)^{\alpha},
$$

and this completes the proof.

Hence, as an immediate corollary we get Dyakonov results for quasiconformal mappings:

Theorem Dy. Suppose $\Omega$ is a $\Lambda_{\alpha}$-extension domain, $0<\alpha \leq 1$, and $f$ is a $K$-quasiconformal mapping of $\Omega$ onto $f(\Omega) \subset \mathbf{R}^{n}$. The following two conditions are equivalent:

a) $f \in \Lambda_{\alpha}(\Omega)$,

b) $|f| \in \Lambda_{\alpha}(\Omega)$.

If, in addition, $\Omega$ is a uniform domain and if $\alpha \leq K^{1 /(1-n)}$, then these conditions are equivalent to

c) $|f| \in \operatorname{loc} \Lambda_{\alpha}(\Omega)$.

\section{References}

[1] Arsenović, M., V. Božin, and V. Manojlović: Moduli of continuity of harmonic quasireglur mappings in $\mathbf{B}^{n}$. - Potential Anal. 34:3, 2011, 283-291.

[2] Arsenović, M., V. Kojić, and M. Matelujević: On Lipschitz continuity of harmonic quasiregular maps on the unit ball in $\mathbf{R}^{n}$. - Ann. Acad. Sci. Fenn. Math. 33:1, 2008, 315318.

[3] Arsenović, M., V. Manojlović, and R. NÄKki: Boundary modulus of continuity and quasiconformal mappings. - Ann. Acad. Sci. Fenn. Math. 37:1, 2012, 107-118. 
[4] Astala, K., and Gehring, F. W.: Quasiconformal analogues of theorems of Koebe and Hardy-Littlewood. - Michigan Math. J. 32, 1985, 99-107.

[5] Caffarelli, L. A., and D. Kinderlehrer: Potential methods in variational inequalities. J. Anal. Math. 37, 1980, 285-295.

[6] Dyakonov, K. M.: Holomorphic functions and quasiconformal mappings with smooth moduli. - Adv. Math. 187, 2004, 146-172.

[7] Gehring, F. W.: Rings and quasiconformal mappings in space. - Trans. Amer. Math. Soc. 103, 1962, 353-393.

[8] Gehring, F. W., and O. Martio: Lipschitz-classes and quasiconformal mappings. - Ann. Acad. Sci. Fenn. Ser. A I Math. 10, 1985, 203-219.

[9] Gilbarg, D., and N. S. Trudinger: Elliptic partial differential equations of second order. Second edition. - Springer, 1983.

[10] Hinkmanen, A.: Modulus of continuity of harmonic functions. - J. Anal. Math. 50, 1988, 1-29.

[11] KalaJ, D.: Lipschitz spaces and harmonic mappings. - Ann. Acad. Sci. Fenn. Math. 34, 2009, $475-485$.

[12] KalaJ, D.: A priori estimate of gradient of a solution to certain differential inequality and quasiconformal mappings. - J. Anal. Math. 118:5, 2012, 1-23.

[13] Kalaj, D., and V. Manojlovic: Subharmonicity of the modulus of quasiregular harmonic. - J. Math. Anal. Appl. 381:2, 2011, 1-6.

[14] KIM, K.: Hardy-Littlewood property with the inner length metric. - Comm. Korean Math. Soc. 19:1, 2004, 53-62.

[15] KoJić, V., and M. Pavlović: Subharmonicity of $|f|^{p}$ for quasiregular harmonic functions with applications. - J. Math. Anal. Appl. 342, 2008, 742-746.

[16] Krantz, S. G.: Function theory of several complex variables. First edition. - John Wiley and Sons, New York, 1982.

[17] Krantz, S. G.: Calculation and estimation of the Poisson kernel. - J. Math. Anal. Appl. 302, $2005,143-148$.

[18] Krantz, S. G.: Some new thoughts on maximal functions and Poisson integral. - J. Math. Anal. Appl. 302, 2010, 143-148.

[19] Lappalainen, V.: Lip $h_{h}$-extension domains. - Ann. Acad. Sci. Fenn. Ser. A I Math. Diss. 56, 1985.

[20] Martio, O.: On harmonic quasiconformal mappings. - Ann. Acad. Sci. Fenn. A I Math. 425, $1968,3-10$.

[21] Martio, O., and R. NÄKki: Boundary Hölder continuity and quasiconformal mappings. - J. London Math. Soc. (2) 44, 1991, 339-350.

[22] Mateljević, M.: Quasiconformality of harmonic mappings between Jordan domains. - Filomat $26: 3,2012,479-510$.

[23] Mateljević, M., and M. Vuorinen: On uniform continuity of harmonic maps. - J. Inequal. Appl. 2010, Article ID 178732.

[24] Nolder, C. A.: A quasiregular analogue of a theorem of Hardy and Littlewood. - Trans. Amer. Math. Soc. 331, 1992, 215-226.

[25] Stein, E. M.: Boundary behavior of holomorphic functions of several complex variables. Princeton Univ. Press, Princeton, 1972.

[26] VÄIsÄLÄ, J.: Lectures on n-dimensional quasiconformal mappings. - Lecture Notes in Math. 229, Springer-Verlag, 1971. 